\title{
Cutting edge of an aggressive surgical approach for perihilar cholangiocarcinoma
}

\author{
Masato Nagino
}

Published online: 5 March 2013

(C) Springer-Verlag Italia 2013

\section{Introduction}

Perihilar cholangiocarcinoma is the most common type of cholangiocarcinoma, accounting for approximately $60 \%$ of cases, followed by the distal and then the intrahepatic forms. Although its surgical resection is technically demanding, many surgeons have adopted an aggressive approach to perihilar cholangiocarcinoma, as surgical resection is the only way to cure this intractable disease. In the past two decades, with advances in diagnostic and surgical techniques, the surgical outcomes and survival rates have gradually improved [1]. In this Editorial, we discuss the benefit of an aggressive surgical approach to perihilar cholangiocarcinoma, including combined vascular resection and hepatopancreatoduodenectomy.

\section{Portal vein resection}

Portal vein invasion was previously the main cause of unresectability of perihilar cholangiocarcinoma. In 1990s, several authors stressed the importance of using portography to demonstrate portal vein invasion as a contraindication to surgery. However, this philosophy has recently become outdated. Combined portal vein resection is now a routine procedure in leading centers in both the East and West, and its clinical benefit has been validated by many studies. Portal vein resection is performed primarily in cases of right-sided hepatectomy, most likely due to

\section{Nagino $(\bowtie)$}

Division of Surgical Oncology, Department of Surgery,

Nagoya University Graduate School of Medicine, 65 Tsurumai-cho, Showa-ku, Nagoya 466-8550, Japan e-mail: nagino@med.nagoya-u.ac.jp technical feasibility. However, we should note that vessel resection is often required in cases of left-sided hepatectomy for advanced tumors with left-sided predominance [2].

In our previous study [3], we reported a mortality of $9.6 \%$ and a 5-year survival rate of $9.9 \%$ in combined liver and portal vein resection for perihilar cholangiocarcinoma ( $n=52$; study period from 1979 to 2000). Since then, the outcome has markedly improved as we have gained more experience: in 185 patients between 2001 and 2012 (portal vein resection alone in 111 patients, simultaneous resection of the portal vein and hepatic artery in 74 patients), the mortality decreased to $3.2 \%$ and the 5-year survival rate increased to $24.6 \%$ (unpublished data). Indeed, 20 patients survived for more than 5 years.

Portal vein resection should be performed only when the vessel adheres to and cannot be freed from the tumor during the skeletonization resection of the hepatoduodenal ligament. We have never used the "no-touch technique" introduced by Neuhaus et al. [4], as it lacks scientific validation. In their series, the 5-year survival rate of $60 \%$ was calculated from the data of only 14 patients, after excluding both operative deaths and $\mathrm{R} 1 / 2$ resections. The mortality in patients who underwent hepatectomy with portal vein resection was as high as $17 \%$. In addition, cancer invasion of the resected portal vein was histologically observed in only $22 \%$ of patients who underwent combined resection. Under the concept of "no-touch technique", these findings strongly indicate that unnecessary portal vein resection was performed in many patients. In his series in which right-sided hepatectomy was preferred, the overall 5-year survival rate including all deaths was only $22 \%$. Although the no-touch technique may warrant further study, we do not believe that these findings demonstrate superiority of the concept. 


\section{Hepatic artery resection}

Recent advances in surgical techniques and knowledge, which have been gained from experiences with liver transplantation, have facilitated the performance of hepatic artery resection with reconstruction. These advances have encouraged some aggressive hepatobiliary surgeons to perform this difficult resection for locally advanced tumors. Most of the previous studies, however, showed negative results and did not recommend the combined resection of the hepatic artery for biliary cancer. For example, Miyazaki et al. [5] reported a mortality rate of $33.3 \%(=3 / 9)$ in patients who underwent hepatectomy with hepatic artery resection, and none of these patients survived for more than 3 years. From these observations, they concluded that hepatic artery resection, unlike portal vein resection, cannot be justified.

In 2010, we reported our experiences with major hepatectomies with simultaneous resections and reconstructions of the portal vein and hepatic artery $(n=50)$ and showed that this challenging surgery can be performed with an acceptable mortality rate of $2 \%$ and offers a better chance of long-term survival, with a 5-year survival rate of $30 \%$ [2]. Thereafter, the number of patients who underwent combined hepatic artery resection for perihilar cholangiocarcinoma has increased, now reaching 107 (including the 50 patients mentioned above). Of them, 28 patients underwent hepatic artery resection alone and the remaining 79 patients underwent simultaneous resection of the portal vein and hepatic artery. The types of hepatectomy performed included left trisectionectomy in 64 patients, left hemihepatectomy in 38, right-sided hepatectomy in 4 , and central bisegmentectomy in 1 . The resected hepatic arteries were reconstructed mainly by end-to-end anastomosis: an interposition graft using the radial artery or greater saphenous vein was required in 18 patients, and an arterioportal shunt was performed in 4 patients, due to the failure of reconstruction. The overall mortality rate was $2.8 \%$, and the 5-year survival rate was $34.1 \%$ (unpublished data).

The clinical significance of hepatic artery resection is still debatable, and further follow-up studies are needed in some leading centers. However, we believe that our data are promising and encourages hepatobiliary surgeons to perform this technically demanding resection.

\section{Hepatopancreatoduodenectomy (HPD)}

A major hepatectomy combined with a pancreatoduodenectomy, i.e., a hepatopancreatoduodenectomy (HPD), is indicated for laterally advanced cholangiocarcinoma. Since Takasaki et al. [6] first introduced HPD for the treatment of locally advanced gallbladder cancer in 1980, many Japanese

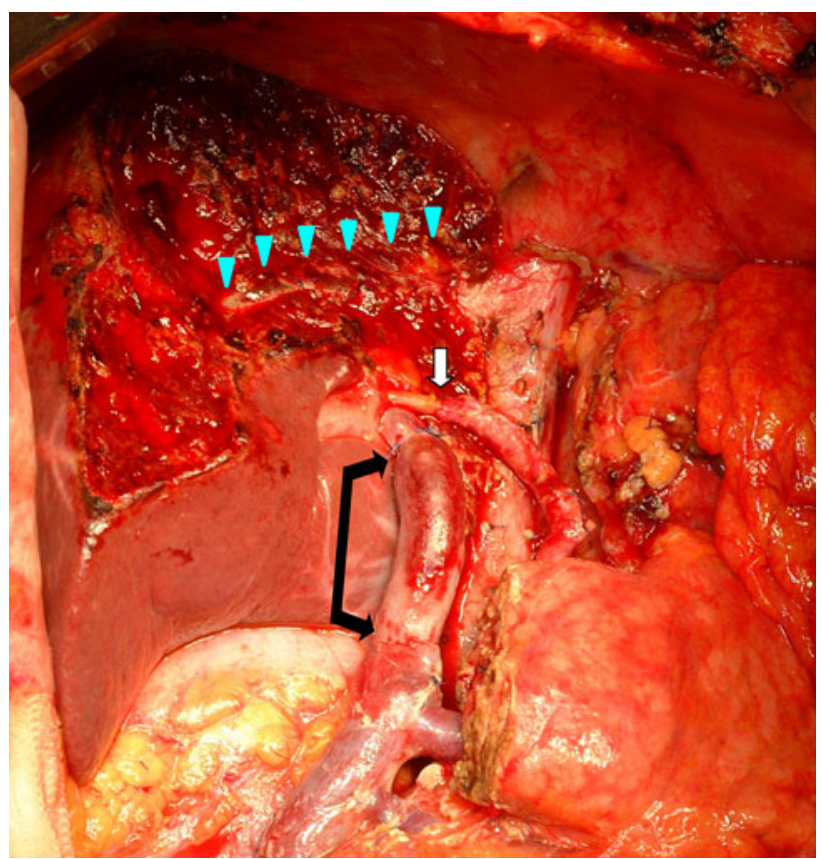

Fig. 1 Completion photograph of hepato-ligamento-pancreatoduodenectomy (HLPD). Left trisectionectomy with pancreatoduodenectomy was performed. The portal vein was reconstructed with external iliac vein interposition graft (black arrow), and the right posterior hepatic artery was reconstructed using the rotating left gastric artery (white arrow). Right hepatic vein (blue arrow heads) was exposed on the transected plane (color figure online)

surgeons and a small number of Western surgeons have employed this extended procedure for the treatment of biliary cancer. However, a limited number of patients and a high rate of mortality ranging from 13 to $60 \%$ hindered our ability to reach to a definitive conclusion regarding the survival benefit of this procedure. Even today, HPD for biliary cancer remains controversial and is the most challenging operation employed by hepatobiliary surgeons.

In 2012, we reported the surgical outcome of 85 patients with cholangiocarcinoma who underwent HPD from 1992 to 2011 [7]. The main tumor location was perihilar in 59 patients and distal in 26 patients. The types of hepatectomy performed included right-sided hepatectomy in 55 patients, left-sided hepatectomy in 23 patients, and central hepatectomy in 7 patients. Combined portal vein resection was performed in 24 patients, and hepatic artery resection was performed in 7 patients. Despite this aggressiveness, the mortality rate was only $2.4 \%$, and the overall 5 -year survival rate was $37.4 \%$. The survival rate of 57 patients with pM0 disease who underwent R0 resection was $54.3 \%$.

Our data clearly demonstrate that HPD can be performed with a low-mortality rate and offers an improved chance of long-term survival in selected patients. We stress that HPD is an important procedure for treating extensively spreading cholangiocarcinoma that is otherwise unresectable, and it is 
now the fourth standard procedure following hepatectomy, bile duct resection, and pancreatoduodenectomy [7].

The current challenge is super-extended resection, i.e., hepato-ligamento-pancreatoduodenectomy (HLPD), in which HPD is performed with an en bloc resection of the hepatoduodenal ligament requiring the simultaneous resection of the portal vein and hepatic artery (Fig. 1). Till date, we have performed this super-extended surgery in 7 patients, without mortality. We will report its outcome and survival in the near future.

\section{Closing remarks}

There are still several issues to be resolved in the surgical treatment of perihilar cholangiocarcinoma; therefore, further collaboration of endoscopists, radiologists, oncologists, and surgeons is essential to conquer this disease. Compared to pancreatic or gallbladder cancer, however, cholangiocarcinoma has rather mild biologic behavior, indicating that the benefit arising from an aggressive surgical approach is great. As chemotherapy is less effective, the most important matter is to relentlessly pursue the possibility of surgical resection. In this regard, hepatobiliary surgeons must make an effort to expand understanding of liver anatomy and to refine their surgical skill, to perform difficult hepatectomies.
Conflict of interest None.

\section{References}

1. Nagino M, Ebata T, Yokoyama $Y$, Igami T, Sugawara G, Takahashi Y et al (2013) Evolution of surgical treatment for perihilar cholangiocarcinoma: A single center 34-year review of 574 consecutive resections. Ann Surg (in press)

2. Nagino M, Nimura Y, Nishio H, Ebata T, Igami T, Matsushita M et al (2010) Hepatectomy with simultaneous resection of the portal vein and hepatic artery for advanced perihilar cholangiocarcinoma: an audit of 50 consecutive cases. Ann Surg 252:115-123

3. Ebata T, Nagino M, Kamiya J, Uesaka K, Nagasaka T, Nimura Y (2003) Hepatectomy with portal vein resection for hilar cholangiocarcinoma: audit of 52 consecutive cases. Ann Surg 238: 720-727

4. Neuhaus P, Jonas S, Bechstein WO, Lohmann R, Radke C, Kling $\mathrm{N}$ et al (1999) Extended resections for hilar cholangiocarcinoma. Ann Surg 230:808-819

5. Miyazaki M, Kato A, Ito H, Kimura F, Shimizu H, Ohtsuka M et al (2007) Combined vascular resection in operative resection for hilar cholangiocarcinoma: does it work or not? Surgery 141:581-588

6. Takasaki K, Kobayashi S, Mutoh H, Akimoto S, Toda K, Asado S et al (1980) Our experiences (5 cases) of extended right lobectomy combined with pancreatoduodenectomy for the carcinoma of the gall bladder. Tan to Sui 1:923-932 (in Japanese)

7. Ebata T, Yokoyama Y, Igami T, Sugawara G, Takahashi Y, Nimura $\mathrm{Y}$ et al (2012) Hepatopancreatoduodenectomy for cholangiocarcinoma: a single center review of 85 consecutive patients. Ann Surg 256:297-305 